\title{
Domenico Felice, avec la collaboration de Giovanni Cristani, Pour l'histoire de la réception de Montesquieu en Italie (1789-2005)
}

\section{Franco Piva}

\author{
(2) OpenEdition \\ Journals \\ Edizione digitale \\ URL: http://journals.openedition.org/studifrancesi/9552 \\ DOI: 10.4000/studifrancesi.9552 \\ ISSN: 2421-5856

\section{Editore} \\ Rosenberg \& Sellier
}

\section{Edizione cartacea}

Data di pubblicazione: 1 décembre 2007

Paginazione: 661

ISSN: 0039-2944

\section{Notizia bibliografica digitale}

Franco Piva, «Domenico Felice, avec la collaboration de Giovanni Cristani, Pour l'histoire de la réception de Montesquieu en Italie (1789-2005)», Studi Francesi [Online], 153 (LI | III) | 2007, online dal 30 novembre 2015, consultato il 08 janvier 2021. URL: http://journals.openedition.org/studifrancesi/9552 ; DOI: https://doi.org/10.4000/studifrancesi.9552

Questo documento è stato generato automaticamente il 8 janvier 2021.

\section{cc) (†) $\odot$}

Studi Francesi è distribuita con Licenza Creative Commons Attribuzione - Non commerciale - Non opere derivate 4.0 Internazionale. 


\title{
Domenico Felice, avec la collaboration de Giovanni Cristani, Pour l'histoire de la réception de Montesquieu en Italie (1789-2005)
}

\author{
Franco Piva
}

\section{NOTIZIA}

DOMENICO FELICE, avec la collaboration de GIOVANNI CRISTANI, Pour l'histoire de la réception de Montesquieu en Italie (1789-2005), Bologna, CLUEB, 2006, pp. 332.

Domenico Felice ha cominciato a interessarsi alla fortuna di Montesquieu in Italia molti anni fa: il suo primo tentativo di ritracciare la storia del fecondo rapporto che la cultura italiana ha intrattenuto fin dal Settecento con l'opera e le idee di Montesquieu risale a oltre vent'anni fa: il suo Montesquieu in Italia (1800-1985) è infatti apparso nel 1986. Nel 1991 al suo Pour l'histoire de la fortune de Montesquieu en Italie (1789-1945), Bologna 1990, che riprendeva e approfondiva le precedenti ricerche, è stato attribuito il Prix Montesquieu. L'indagine è stata ulteriormente approfondita, completata, e portata fino al 1990, cinque anni più tardi nel volume Moderation et justice. Lectures de Montesquieu en Italie, apparso con la prefazione di un altro grande studioso di Montesquieu, Jean Ehrard. Il presente volume riprende la sostanza delle indagini precedenti con l'aggiunta di un capitolo, affidato alle cure di uno degli allievi di D. Felice, Giovanni Cristani, che prende in considerazione gli ultimi 10 anni della fortuna di Montesquieu in Italia: anni particolarmente fecondi grazie, anche se non soprattutto, ai lavori sul pensiero e sull'opera di Montesquieu che Domenico Felice ha condotto in prima persona o coordinato, e che Cristani ha analizzato con molta diligenza e perizia, mettendo bene in luce non solo l'attenzione che gli studiosi italiani hanno portato all'autore delle Considérations e dell'Esprit des lois, ma anche l'assoluta eccellenza degli studi italiani su Montesquieu, dimostrata sia dai riconoscimenti che alcuni studiosi 
italiani hanno ottenuto al di là delle Alpi sia dalla loro inclusione nel gruppo di lavoro che da alcuni anni sta procedendo alla pubblicazione di un'edizione critica delle Opere del barone de la Brède destinata a fare epoca. Un rapporto, quello che la cultura italiana ha intrattenuto e ancora oggi intrattiene con l'opera di Montesquieu, che non solo è risultato fruttuoso a livello di ricerca storica o erudita ma che ha anche influenzato profondamente, e proficuamente, la nostra vita politica e civile, a partire dall'epoca delle Repubbliche sorte al tempo della grande Rivoluzione e fino agli anni, a noi assai vicini, di Mani pulite, passando per il momento, fondamentale per la nostra storia, della Costituzione sulla quale è stata fondata la prima Repubblica, e che proprio da Montesquieu ha tratto spesso ispirazione, in particolare per quell'equilibrio tra $\mathrm{i}$ diversi poteri su cui i Padri Costituenti, pur nella diversità delle opinioni politiche e ideologiche, hanno voluto, o cercato, di fondare la Prima Repubblica, e che in tempi recentissimi qualcuno ha tentato di rimettere, almeno in parte, in discussione. Proprio per questo la bella ricostruzione della fortuna di Montesquieu in Italia operata da Domenico Felice e da Giovanni Cristani assume il sapore e il significato di un dibattito anche politico, per di più di una bruciante attualità, che molti avrebbero interesse a ripercorre con attenzione. 